\title{
SISTEMA DE PROGRAMACIÓN Y CONTROL AUTOMÁTICO DE UN RIEGO POR GOTEO SUBTERRÁNEO EN UN CULTIVO DE OLIVAR
}

\author{
Autores: Reca Cardeña, J. (1),Martínez López, J. (1), Sánchez Molina, J.A. (2), Rivas Ruiz, \\ J.L. (2), Ferre Giménez, J.A. (2) \\ ${ }^{1}$ Profesor Titular de Universidad. Universidad de Almería. Departamento de Ingeniería, Crta. \\ Sacramento S.N. La Cañada de S. Urbano. 04120. Almería. jreca@ual.es \\ ${ }^{2}$.Grupo de Investigación en Automática, Electrónica y Robótica. Universidad de Almería. \\ Crta. Sacramento S.N. La Cañada de S. Urbano. 04120. Almería. jorgesanchez@ual.es
}

\section{Resumen}

En esta comunicación se presenta un nuevo sistema de programación y control automático del riego por goteo subterráneo para un cultivo de olivar ecológico de una finca situada en el T.M. de Tabernas en Almería (España) y propiedad de la empresa Rafael Alonso Aguilera S.L. La finca se riega mediante un novedoso sistema de riego subterráneo patentado por esta empresa. El sistema de control propuesto está basado en el establecimiento de un balance hídrico diario en el en el sistema suelo-planta a partir de la estimación de las necesidades de riego del cultivo en tiempo real a partir de los datos climáticos medidos en tiempo real a partir de una estación agroclimática. Se presentan también los resultados de la evaluación del funcionamiento del sistema a lo largo de un periodo de 5 años y se analiza el ahorro de agua proporcionado por la combinación del sistema de control del riego y del método de riego subterráneo.

\section{1- Introducción. Objetivo del trabajo}

Un manejo eficiente del agua de riego es fundamental, especialmente en zonas áridas, como es el caso de Almería, en las que el agua constituye un factor de producción escaso y vulnerable. Las condiciones favorables de radicación y temperatura en esta zona han permitido el desarrollo de cultivos de gran calidad y rentabilidad económica, como es el caso de las recientes plantaciones de olivar en regadío en la zona de Tabernas. No obstante, el manejo inadecuado del riego puede provocar serios impactos ambiéntales como el agravamiento del déficit hídrico, la erosión del suelo y la contaminación de los acuíferos por lixiviación de nutrientes o fitosanitarios.

La utilización de sistemas de riego por goteo subterráneos (Ayars y col., 1999) permite mejorar la eficiencia del riego ya que aplican el agua en el interior del suelo y evitan las pérdidas de agua por evaporación directa. Además, localizan mejor el agua y los nutrientes en la zona donde la absorción radical es más activa, favoreciendo su correcta absorción. Sin embargo, presenta algunos inconvenientes que dificultan su manejo como la rotura de tuberías debido a la acción de las raíces, la obturación de los emisores y la dificultad de localización y reposición de emisores obturados y fugas de agua. Para evitar estos inconvenientes, un nuevo método de riego subterráneo, patentado por Rafael Alonso Aguilera S.L. (en adelante sistema RA), se está utilizando con éxito en la zona. Este sistema, consiste en instalar la red de distribución de agua y los goteros en superficie e introducir la descarga de los goteros en profundidad, a través de unas tuberías perforadas de plástico colocadas verticalmente en el suelo (ver figura 1). Este sistema es sencillo de instalar, económico y potencialmente eficiente, por presentar todas las ventajas del riego subterráneo. 


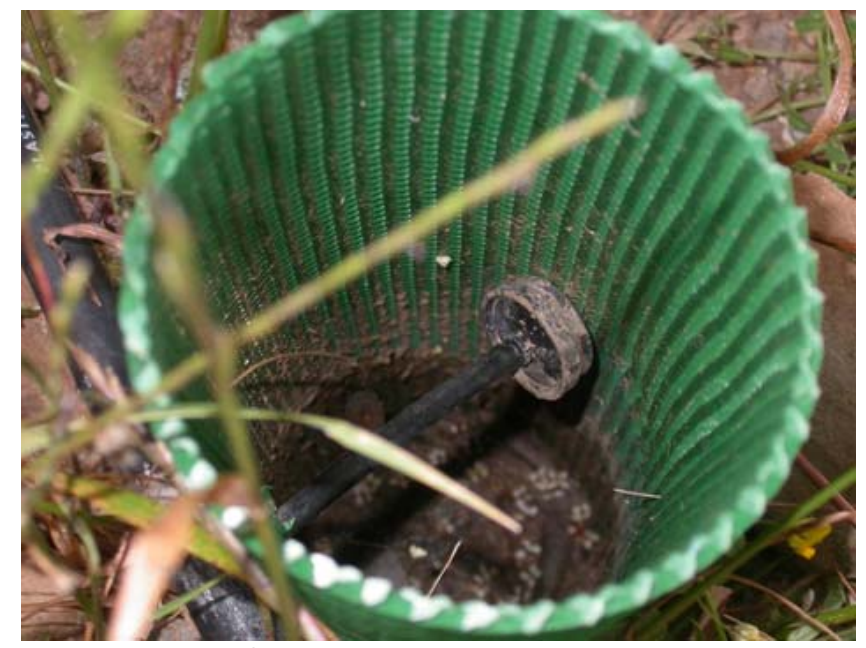

Figura 1. Sistema de riego subterráneo RA

En el año 2005, nuestro grupo inicia un proyecto de investigación financiado por la mencionada empresa, cuyo objetivo fundamental fue evaluar agronómicamente el nuevo sistema de riego por goteo subterráneo en un cultivo de olivar ecológico de la zona de Tabernas. Para ello se planteó un ensayo de campo, en el que se evaluó la respuesta del nuevo sistema de riego RA, en comparación con un sistema de riego por goteo superficial, bajo tres dosis de agua diferentes $(100 \%, 80 \%$ y $60 \%$ de las necesidades de riego respectivamente). Este ensayo de campo se prolongó durante 3 campañas (2005, 2006 y 2007) en una primera fase y 2 campañas más (2009 y 2010) en una segunda fase del proyecto. Los resultados de este estudio experimental demostraron que el sistema de riego RA resultó ser más eficiente ya que permitió mejorar la eficiencia del uso del agua hasta en un 20\% (Martínez y Reca, 2014). No obstante, poder hacer efectivo dicho ahorro, es necesario realizar una programación y un manejo del riego en tiempo real, con criterios racionales que tengan en cuenta las necesidades de riego del cultivo con este nuevo sistema

Como consecuencia, en el año 2009 se inicia un nuevo proyecto, cuyo objetivo fundamental fue desarrollar un sistema automático de programación y control del riego para un cultivo de olivar ecológico regado con el nuevo sistema RA. El objetivo es implementar en el mencionado sistema de control los resultados obtenidos en los ensayos de campo, junto con una metodología racional de cálculo de necesidades hídricas. Esta herramienta permitirá conseguir la máxima eficiencia en el uso del agua de riego con el sistema de riego subterráneo RA, sin que el agricultor tenga que preocuparse de realizar la programación del riego. En este trabajo, se presenta el desarrollo de este nuevo sistema de programación y control automático del riego de un cultivo de olivar ecológico regado con el sistema RA y los resultados del seguimiento de su funcionamiento durante un total de 5 campañas agrícolas.

\section{2- Materiales y métodos.}

Para evaluar el funcionamiento del nuevo sistema de control automático del riego, se desarrolló un prototipo del mismo que se instaló en una finca de olivar ecológico propiedad de Rafael Alonso Aguilera S.L., situada en la comarca del desierto de Tabernas de la provincia de Almería (España).

\subsection{Caracterización de la finca experimental}

La primera actividad que fue necesario realizar para el desarrollo del sistema de control fue la caracterización de la finca de olivar y de su instalación de riego. Dicha 
explotación, tiene una superficie total de cultivo de aproximadamente 56,28 ha, fundamentalmente de olivar ecológico (ver figura 2).

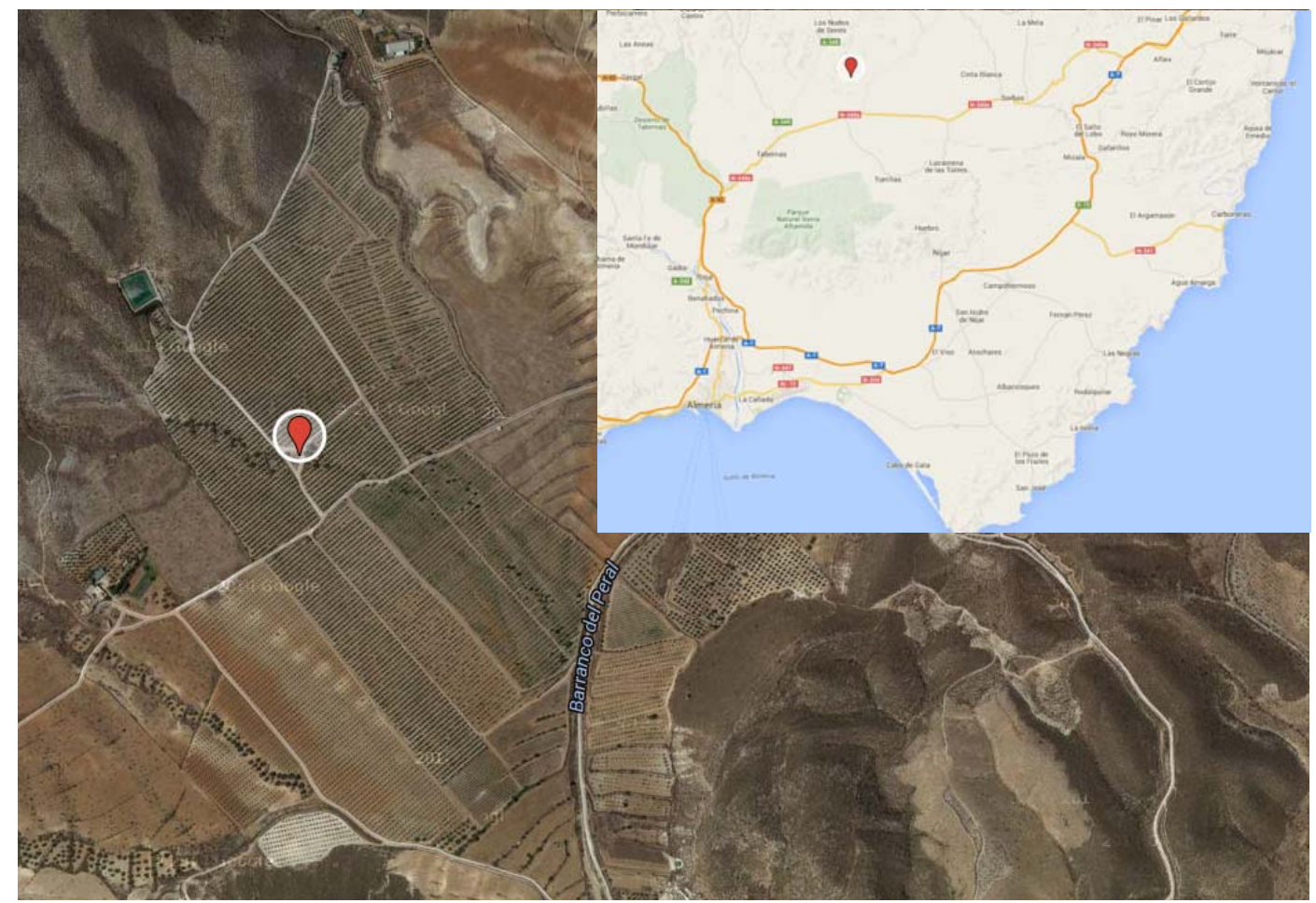

Figura 2. Situación y emplazamiento de la finca experimental

Actualmente dispone de un sistema de riego por goteo subterráneo RA. El agua de riego, procedente del acuífero de la zona, la extrae mediante 4 sondeos, con sus respectivas bombas, que la inyectan directamente a la red de distribución de agua. Esta red presenta dos depósitos de regulación desde los que riega por gravedad. Debido a que los depósitos se encuentran a distinta cota y para evitar que se vacíe el de mayor altura, la red sólo se puede alimentar alternativamente de uno de ellos. Cuando los requerimientos de presión del sistema son altos, se alimenta del depósito situado a mayor cota, mientras que en el resto de ocasiones se alimenta del localizado a menor cota. Esta operación se realiza mediante dos electroválvulas motorizadas que alternativamente abren y cierran los depósitos que correspondan. El método de riego es por goteo subterráneo RA en toda la finca y se utilizan goteros de tipo autocompensante. El número de goteros por árbol y el caudal de cada uno varían entre sectores de riego, dependiendo de la edad y marco de plantación del cultivo. El equipo de filtración está formado por 5 filtros de anillas de 125 micras autolimpiantes. La operación de limpieza se realiza por tiempo y por variación de presión entre la entrada y la salida del filtro para lo que se dispone de un manómetro diferencial tarado para activar el sistema de limpieza cuando dicha presión diferencial sea mayor que un valor prefijado. El equipo de fertirriego está formado por 3 bombas dosificadoras, cuatro depósitos de fertilizante y dos agitadores neumáticos.

Para el diseño del sistema de control se han establecido ocho sectores independientes de riego con diferentes variedades, edades, marcos de plantación y número y caudal de goteros.

\subsection{Balance hídrico en el sistema suelo-planta.}

El sistema de programación automática implementado se basa en el establecimiento de un balance hídrico diario. En un sistema suelo-planta, bien delimitado y considerando las 
entradas y salidas a dicho sistema, puede plantearse la siguiente ecuación de balance hídrico o conservación de masa (ecuación 1):

$$
A_{t}=A_{t-1}+H_{n}+P e-E T_{c}
$$

Donde: $A_{t}$ y $A_{t-1}$ son el agotamiento para un día $t$ y $t-1$, respectivamente, $H_{n}$ es la lámina neta de riego, $P_{e}$ la precipitación efectiva o fracción de la precipitación total que es utilizada por el cultivo, $E T_{c}$ es la evapotranspiración del cultivo o agua que se pierde por evaporación desde el sistema suelo-planta.

La lámina neta de riego se calcula a partir de la lámina bruta $\left(H_{b}\right)$ y del rendimiento de aplicación $\left(R_{a}\right)$ del riego que tiene en cuenta las pérdidas de agua en la aplicación.

$$
H_{n}=\frac{H_{b}}{R_{a}}
$$

La lámina bruta $\left(H_{b}\right.$ en $\left.\mathrm{mm}\right)$ aportada en cada riego se estima en función del tiempo de aplicación del riego $\left(t_{a r}\right.$, en $\left.\mathrm{h}\right)$, del número de goteros por árbol $\left(n_{e}\right)$, del caudal del emisor ( $q$ en $\mathrm{I} / \mathrm{h}$ ) y del marco de plantación en $\mathrm{m}^{2}\left(s_{a} s_{f}\right)$.

$$
H_{b}==\frac{n_{e} \cdot q \cdot t_{a r}}{s_{a} \cdot s_{f}}
$$

La precipitación efectiva calcula a partir de la precipitación medida mediante un pluviómetro y con la aplicación de un coeficiente reductor (Dastane, 1978).

La evapotranspiración del cultivo se puede estimar a partir de la medida de variables climáticas siguiendo la metodología propuesta por la FAO (Allen et al., 1998). Según esta metodología, la $E T_{c}$ se calcula como el producto de 3 términos:

$$
E T_{c}=E T_{o} \cdot K_{c} \cdot K_{r}
$$

Donde: $E T_{o}$ es la evapotranspiración de referencia, $K_{c}$ es el coeficiente de cultivo y $K_{r}$ es un coeficiente reductor por localización.

La evapotranspiración de referencia $\left(E T_{0}\right)$ se ha calculado mediante la ecuación de Penman-Monteith (Allen y col. 1998). En este trabajo se han utilizado los coeficientes de cultivo mensuales propuestos por Orgaz y Fereres (1999). Para el coeficiente reductor por localización $\left(K_{r}\right)$ se ha utilizado la fórmula propuesta por Fereres et al. (1981). Debido a que el sistema de riego era un nuevo sistema de riego subterráneo, se ha introducido también un factor reductor para tener en cuenta la reducción de evaporación desde el bulbo húmedo. El valor recomendado para este factor reductor oscila entre fue estudiado experimentalmente por el equipo investigador en un estudio previo realizado (Martínez y Reca, 2014) y se recomiendan valores que oscilan entre 0.8 y 0.9 .

\subsection{Criterios de programación del riego.}

La programación del riego está basada en la aplicación de dosis de riego fijas y en intervalos de riego variables dependiendo de las necesidades de riego del cultivo.

El cálculo de las dosis de riego se ha realizado tomando el valor de la $E T_{c}$ en la época de máximo consumo para un olivar con un grado de cobertura superficial completa. Los tiempos de riego de cada sector se han calculado aplicando la ecuación 4, redondeándolos posteriormente a múltiplos de $1 \mathrm{~h}$. Con estos criterios se han obtenido los tiempos de riego y las dosis que se muestran en la tabla 1 para cada uno de los sectores de riego. 
Estos cálculos se realizan de forma automática por el sistema de control en función de los parámetros que caracterizan al cultivo y al sistema de riego de cada sector (variedad, marco de plantación, caudal por árbol y diámetro de copa).

Tabla 1. Características de los sectores de riego, tiempos de riego y dosis por árbol.

\begin{tabular}{|c|c|c|c|c|c|c|c|c|}
\hline Sector & Variedad & Plant. & $\begin{array}{c}\text { Marco } \\
\left(\mathrm{m}^{2}\right) \\
\end{array}$ & Sup. (ha) & $\begin{array}{c}\text { Q árbol } \\
\text { (I/h) }\end{array}$ & $\begin{array}{l}\text { D. Copa } \\
(\mathrm{m})\end{array}$ & $\begin{array}{l}T_{\text {ar }} \\
\text { (h) }\end{array}$ & $\begin{array}{c}\text { Dosis } \\
\text { (l/árbol) }\end{array}$ \\
\hline 1 & Picual & 2009 & $7 \times 5$ & 7.77 & 4.00 & 1.02 & 1 & 4.00 \\
\hline 2 & Arbequina & 2002 & $7 \times 5$ & 6.11 & 15.67 & 3.08 & 3 & 47.01 \\
\hline 3 & Arbequina & 2002 & $7 \times 5$ & 7.57 & 15.67 & 3.08 & 3 & 47.01 \\
\hline 4 & Picual & 2005 & $7 \times 5$ & 6.54 & 15.67 & 2.78 & 3 & 47.01 \\
\hline 5 & Picual & 2005 & $7 \times 5$ & 3.22 & 15.67 & 2.78 & 3 & 47.01 \\
\hline 6 & Hojiblanca & 2000 & $7 \times 7$ & 4.70 & 25.00 & 3.96 & 3 & 14.10 \\
\hline 7 & Arbequina & 2007 & $7 \times 1.2$ & 7.32 & 4.60 & 1.32 & 2 & 9.20 \\
\hline 8 & Picual & 2007 & $7 \times 5$ & 11.06 & 6.30 & 1.56 & 2 & 12.60 \\
\hline
\end{tabular}

\subsection{Descripción del sistema de control}

Los elementos físicos que componen el sistema de control son los siguientes:

1. Estación agroclimática: es la encargada de medir las variables climáticas necesarias para el cálculo de la evapotranspiración del cultivo y de la precipitación efectiva (ver figura 3). La estación está dotada de los siguientes sensores (Campbell Scientific):

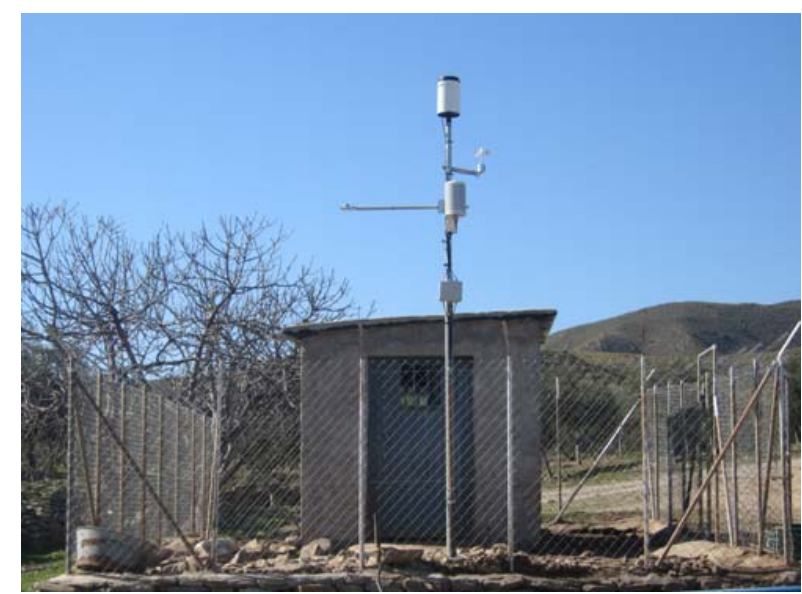

Figura 3. Estación agroclimática instalada en la finca experimental.

- Sensor de temperatura y humedad relativa modelo HMP45C. La temperatura se mide con un sensor Pt1000, que se basa en la variación de la resistencia del platino. La humedad se mide con un dispositivo capacitivo de estado sólido. Ambos sensores se alojan en el interior de una garita modelo 41003-5.

- Anemómetro modelo A100L2. Es un sensor de tres cazoletas preciso y de bajo consumo. El anemómetro está fabricado con una aleación de aluminio anodizado, acero inoxidable y plásticos resistentes al clima.

- Piranómetro de silicio modelo CS300, fabricado por Apogee Instruments, usa un detector fotovoltaico de silicio. 
- Pluviómetro RMYoung 52202. Es un sensor de balancín con sendas cazoletas situadas en sus extremos. Cuando se llena la cazoleta superior, el balancín bascula y emite un pulso. Cada vuelco del brazo equivale a $0.1 \mathrm{~mm}$.

2. Autómata programable, modelo Compact FieldPoint, comercializado por la empresa National Instruments ( $\mathrm{NI}$ ), encargado de controlar todos los elementos del sistema de riego (sensores y actuadores) y dar robustez al sistema. Se trata de una nueva familia de controladores, que se denominan Controladores de Automatización Programables (PACs), que supera en prestaciones a los tradicionales Controladores Lógicos Programables (PLCs). Los PACs de National Instruments están basados en la tecnología LabVIEW de NI. Con LabVIEW, se pueden crear sistemas de medición y control a la medida. El sistema permite integrar subrutinas programadas en Mathlab. Este automáta programable está dotado también de módulos de entradas y salidas de datos tanto analógicos como digitales.

3. Ordenador industrial, modelo IPPC-6152A, comercializado por Advantech. Es el encargado de registrar las variables climáticas, realizar el cálculo de las necesidades hídricas en tiempo real, establecer la programación de riegos más racional e introducirla en el autómata programable. Este equipo posee una pantalla táctil que permite una comunicación más versátil e interactiva entre el usuario y el sistema de control el encargado de servir de interfaz de usuario, también permite su conexión a través de Internet con otro equipo remoto. Las características técnicas de este equipo son las siguientes: procesador Socket 478 Celeron M $1.5 \mathrm{GHz}$, memoria 1 GB DDR2 240pin SDRAM 400/533 MHz, disco duro HDD 80GB SATA-300 2.5" SEAGATE y pantalla táctil XGA TFT LC 15

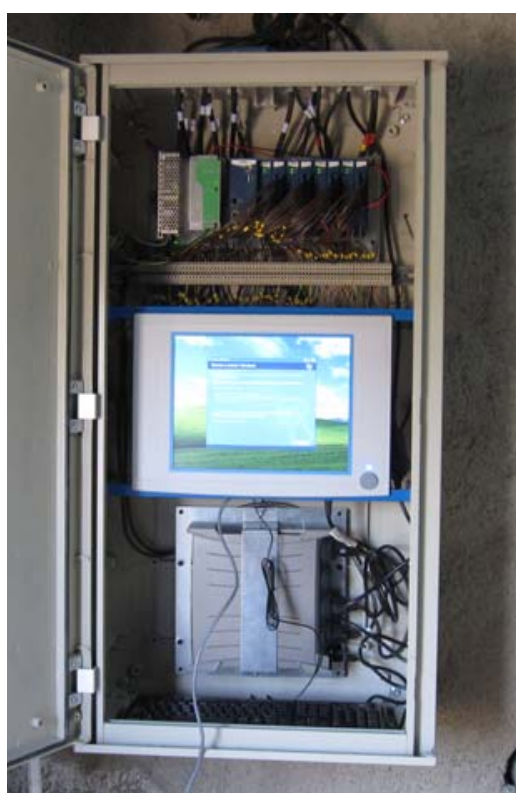

Figura 4. Armario de control con el autómata programable, ordenador industrial y sistema de alimentación ininterrumpida (SAI).

El ordenador industrial y el autómata programable se han instalado en el interior de un armario de protección situado dentro de la caseta que alberga la cabeza del sistema de riego. En dicho armario se encuentra el Sistema de Alimentación Ininterrumpida (SAI), que alimenta a ambos equipos y los protege de sobretensiones. Además, el armario contiene dos fuentes de alimentación, una de 24 VDC y 5A, que alimenta al controlador, y al modulo de entradas analógico (sensores climáticos y transductores de presión), y otra de 12 VDC y $5 \mathrm{~A}$, que alimenta los módulos de salidas digitales (relés de actuación) (ver figura 4).

\section{3- Resultados y conclusiones.}


Se ha desarrollado una aplicación informática original denominada SISRIERA que permite controlar los distintos elementos que componen el sistema de riego (sistemas de bombeo, depósitos, sectores de riego y sistemas de abonado y filtrado), ejecutar los algoritmos de cálculo de necesidades y de balance hídrico, adoptar las decisiones de riego más adecuadas y ejecutar dichas ordenes, organizando y priorizando el riego de los distintos sectores de la explotación y todo ello, a través de una interfaz plenamente amigable e interactiva. Para permitir su adaptación a otros tipos de explotaciones y su extensión a otros cultivos, el sistema se ha diseñado con elementos y protocolos de comunicación abiertos, con objeto de que sea perfectamente configurable y escalable. La conexión del ordenador personal a Internet, con ayuda del escritorio remoto, permite disponer de todos los datos y actuar sobre el sistema de forma remota desde cualquier otro ordenador personal con conexión a Internet.

La pantalla principal de la aplicación de control se muestra en la figura 5. A esta pantalla se puede acceder localmente desde el ordenador industrial instalado en la caseta de riego o por vía remota. En este segundo caso, es necesario especificar un nombre de usuario o una clave para acceder al sistema.

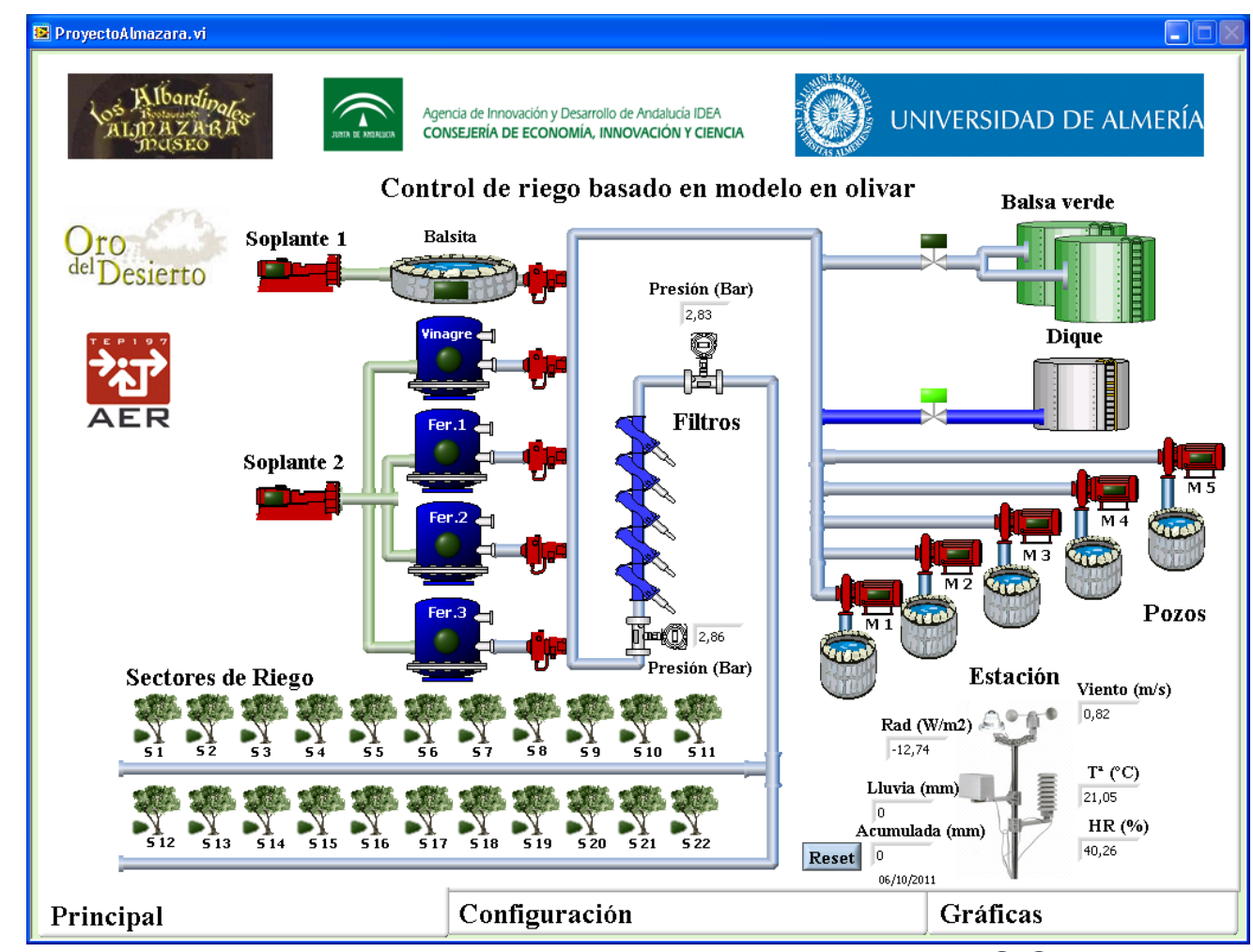

Figura 5. Pantalla principal del sistema de control SISRIERA

La pantalla "Principal" muestra de forma visual el estado de todos los elementos que se pueden controlar en el sistema, además de las variables climáticas a las que se tiene acceso desde la estación meteorológica instalada en la finca.

En la parte inferior derecha de la pantalla se muestra un esquema de la estación climática y la información en tiempo real medida por los sensores: Humedad Relativa (HR, $\%)$, Temperatura $\left(\mathrm{T}^{\mathrm{a}},{ }^{\circ} \mathrm{C}\right)$, Viento $(\mathrm{m} / \mathrm{s})$, Radiación Solar $(\mathrm{Rad}, \mathrm{W} / \mathrm{m} 2)$, Precipitación diaria (Lluvia, mm) y Precipitación Acumulada (Acumulada, mm). La lluvia acumulada puede inicializarse mediante el botón "Reset" que está situado a su izquierda y el texto inferior indica la fecha del última reinicio 
Por encima de la estación meteorológica se muestran los iconos de 5 motores que representan a los grupos motobomba que inyectan el agua en la red de distribución. En la esquina superior derecha de la pantalla se muestran los dos depósitos elevados que alimentan al sistema de riego. La balsa verde, que se encuentra situada a mayor cota, se utiliza para regar los sectores con mayores requerimientos de presión, mientras que el dique se utiliza en el resto de los casos. El sistema debe evitar la apertura simultánea de ambos depósitos. En la parte superior izquierda se muestra el sistema de abonado. Éste consta de 5 bombas inyectoras con sus correspondientes depósitos de fertilizante. Para la agitación de las soluciones nutritivas el sistema dispone de 2 bombas soplantes. A continuación se encuentra el sistema de filtrado de agua que consta de un grupo de 5 filtros de anillas autolimpiantes dispuestos en paralelo. Finalmente, se muestran las unidades de riego (sectores), representados cada una de ellas mediante un olivo. Todos los actuadores (electroválvulas, bombas, etc.) disponen de un indicador (led) que se ilumina en caso de que dicho elemento esté en funcionamiento.

La pestaña "Configuración" permite acceder y editar todos los parámetros configurables del sistema. Entre ellos se pueden destacar los siguientes:

- Modo de funcionamiento: el sistema admite un modo de funcionamiento completamente automático en el que el se adoptan y ejecutan las decisiones de riego sin intervención alguna del usuario y un modo semiautomático en el que el usuario tiene que definir los programas de riego deseados manualmente. Este segundo modo de funcionamiento es equivalente a los sistemas de automatización del riego más habituales.

- $\quad$ Sensores: permite configurar la recta de calibración de los sensores y ver el estado de las salidas digitales que actúan sobre los relés.

- $\quad$ Planificación de riegos: se visualizan los riegos previstos para la semana en el modo semiautomático: hora de inicio, hora final, abonado, etc.

Riego manual: esta opción permite configurar los riegos semanales de forma manual. Se pueden definir las horas de comienzo y finalización del riego, el plan de abonado, el orden de riego de los sectores, etc.

- Riego automático: en esta opción se configura el modo de funcionamiento automático. En este caso no es necesario definir la planificación de riego ya que éstos se calculan de forma automática por el algoritmo de balance de agua. Sólo es necesario configurar las opciones de abonado.

- Motores: esta opción permite configurar los programas de puesta en marcha y parada de los diferentes sondeos así como la apertura y cierra de las llaves de paso de los embalses de acumulación.

- Soplantes: Permite la configuración de los soplantes o agitadores neumáticos asociados a cada depósito de abonado.

- $\quad$ Limpieza filtros: Permite configurar las opciones disponibles para realizar la limpieza de los filtros, tanto por diferencia de presión como periódicamente.

- Algoritmo: Contiene los parámetros asociados al algoritmo que calcula la planificación de riegos automáticos. En este apartado se configuran los datos de los sectores de riego, características de la plantación, y parámetros de cálculo de las necesidades y del balance hídrico.

Uno de los aspectos originales del sistema diseñado, además de programar automáticamente el riego de los sectores de forma individual, es la organización de la aplicación de los riegos, dando prioridad a los sectores con mayor déficit hídrico y dejando para el final a los que tienen menores necesidades. De esta forma, los riegos se ordenan dentro de la jornada atendiendo a sus necesidades y, posponiendo el riego de los sectores menos necesitados para otro día, en caso de que el tiempo de riego disponible en la jornada sea insuficiente para regar todos los sectores necesitados.

El diseño e instalación del prototipo experimental situado en la finca propiedad de Rafael Alonso Aguilera S.L se concluyó en el año 2011, estando el sistema operativo desde 
el día 19 de mayo de 2011 hasta la actualidad. Se dispone, por tanto de un periodo de evaluación del sistema de unos 5 años de funcionamiento continuado. Durante este tiempo y tras un periodo inicial de puesta a punto del sistema, éste ha funcionado de forma continua sin apenas interrupciones, salvo algunas debidas a cortes del suministro eléctrico o alguna labor de mantenimiento. Esto hecho confirma que el sistema diseñado ha demostrado su robustez y fiabilidad.

Con objeto de contrastar las condiciones climáticas en la zona de estudio, se han comparado los datos climáticos registrados por la estación climática del sistema de control, con los datos climáticos de la estación climática de Tabernas, perteneciente a la red Agroclimática de la Junta de Andalucía, situada cerca de la finca experimental. Las variables climáticas medidas han correlacionado bastante bien con los datos de la estación de Tabernas. Los coeficientes de correlación han sido del 98\% para la temperatura media, del $94 \%$ para la humedad relativa, del $98 \%$ para la radiación y del $75 \%$ para la velocidad media de viento. En el caso de la velocidad del viento, se midieron valores inferiores en el anemómetro instalado en la finca con respecto al de la estación de Tabernas. Esto es debido a que la finca se encuentra en una zona protegida, rodeada de montañas en la que la incidencia del viento es localmente menor que en la estación de Tabernas que se encuentra más expuesta. Como consecuencia de ello, la $E T_{o}$ calculada por el sistema de control ha resultado menor a la $E T_{o}$ proporcionada por la estación de Tabernas (un $78 \%$ de esta), debido a la influencia menor del viento (ver Figura 6). La medida local de las condiciones climáticas ha permitido calcular mejor las necesidades del cultivo de forma local ahorrando aproximadamente un $22 \%$ respecto a las que se calcularía utilizando los datos de Tabernas.

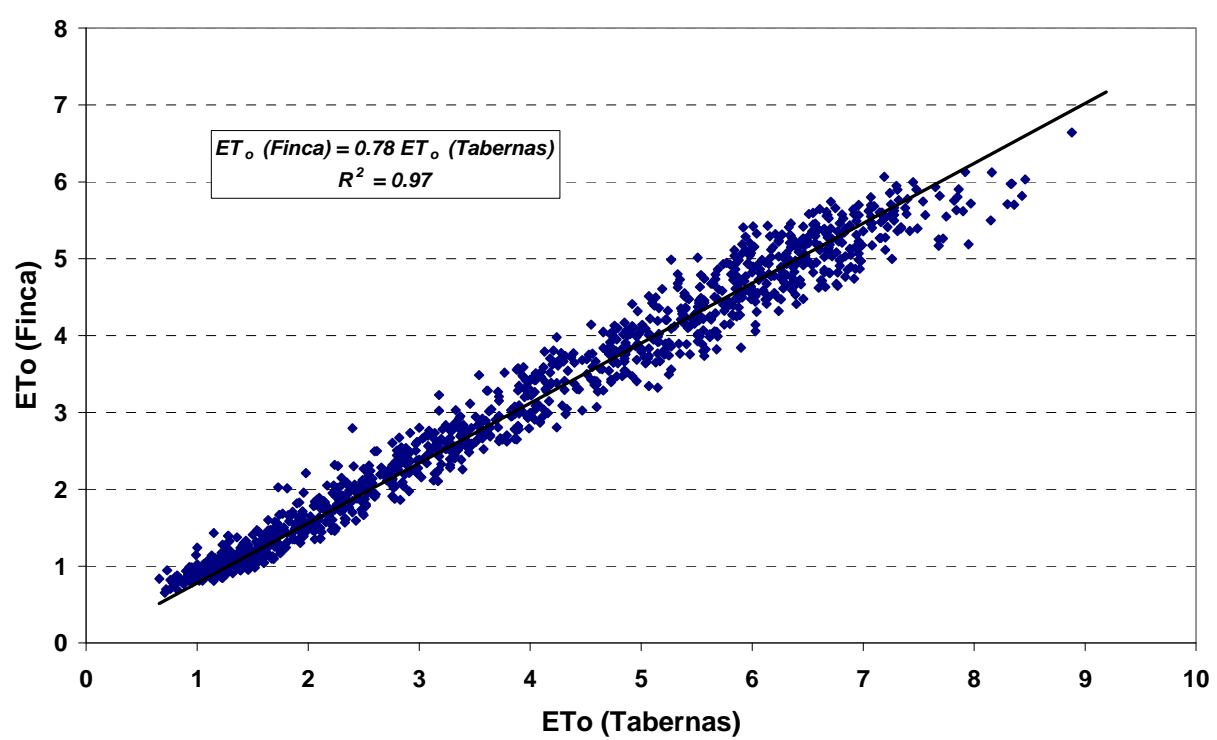

Figura 6. Comparación $E T_{o}$ medida en la finca y en la estación agroclimática de Tabernas (2012-2015)

Durante este periodo se ha comprobado el uso eficiente y racional del agua de riego que este sistema automático ha permitido realizar. Una de sus principales ventajas ha sido la posibilidad de establecer una programación de riego individualizada para cada uno de los 8 sectores de riego homogéneos en los que se ha organizado la explotación. Esto ha permitido ajustar las dosis de riego a las necesidades específicas de cada uno de ellos en función de las variedades, edad del cultivo o marco de plantación. La tabla 1 recoge los riegos totales registrados por el sistema durante un periodo de funcionamiento de 4 años (desde el 1 de enero de 2012 hasta 31 de diciembre de 2015). Durante los tres primeros años del 2012 a 2014, los días fuera de servicio fueron mínimos, sin embargo, durante el 2015 el sistema dejó de funcionar debido a una avería en un sensor y no fue posible su reparación hasta trascurrido un tiempo. En dicha tabla, se recogen además, el número de horas de funcionamiento total del sistema en modo automático y las dosis de riego 
aportados por sector. Se puede comprobar como las dosis de riego anuales han sido variables entre sectores debido a sus diferentes características. Las dosis de riego anuales han oscilado desde una decena de $\mathrm{mm}$ para una plantación joven con un marco amplio hasta valores superiores a los $200 \mathrm{~mm}$ para los sectores con un olivar adulto y con mayor superficie de cubierta vegetal. La dosis media para toda la finca durante el periodo de estudio ha sido de unos $116 \mathrm{~mm}$.

Tabla 1. Riegos totales registrados por el sistema automático durante 4 años de funcionamiento.

\begin{tabular}{|c|c|c|c|c|c|c|c|c|c|c|c|}
\hline \multirow[b]{2}{*}{ Año } & \multirow[b]{2}{*}{ Días } & \multirow{2}{*}{$\begin{array}{c}\text { Riego } \\
\text { (Horas) }\end{array}$} & \multicolumn{9}{|c|}{ Dosis riego (mm/año) } \\
\hline & & & Sec. 1 & ec. 2 & ec. 3 & Sec. 4 & Sec. 5 & Sec. 6 & Sec. 7 & ec. 8 & Media \\
\hline 2012 & 343 & 1781 & 16.8 & 143.7 & .7 & 3.2 & & & 112.2 & 37.4 & 103.6 \\
\hline 2013 & 355 & & 2 & 9.4 & .4 & 8.0 & 0 & .0 & 151.8 & 51.5 & 141.8 \\
\hline 2014 & 363 & & 22. & 4.4 & 194.4 & 134.0 & 13 & 6 & 148.5 & 49.7 & 137.8 \\
\hline 2015 & 296 & 14 & 17.3 & 110.8 & 110.8 & 78.4 & 78.4 & 132.7 & 86.3 & 28.8 & 80.4 \\
\hline edia & 339 & 2005 & 20.0 & 162.1 & 162.1 & 113.4 & 113.4 & 189.7 & 124.7 & 41.9 & 115.9 \\
\hline
\end{tabular}

Como conclusión, cabe destacar que el sistema ha gestionado el riego de una finca de olivar ecológico en producción durante un periodo de tiempo de unos 5 años, de forma eficiente, fiable y autónoma. El sistema de control ha conseguido un ahorro de agua significativa respecto a la gestión del riego anterior (20\% menos de agua aproximadamente), habiendo permitido mantener e incluso incrementar los niveles productivos de la finca, consiguiendo una mejor eficiencia del uso del agua. Como consecuencia de ello, es previsible que este uso más racional del agua si se extiende a otras fincas de la zona, pueda contribuir a garantizar la sostenibilidad de la actividad agrícola en la zona del desierto de Tabernas tan vulnerable a la falta de recursos hídricos.

\section{4.- Agradecimientos}

Los autores agradecen a la empresa Rafael Alonso Aguilera S.L. (Oro del desierto) el apoyo financiero para desarrollar el trabajo de investigación y su iniciativa y entusiasmo.

\section{5- Bibliografía}

Allen, R.G., L.S. Pereira, D. Raes and M. Smith. (1998). Crop evapotranspiration. Guidelines for computing crop water requirements. FAO Irrigation and Drainage Paper 56. FAO. Roma Ayars, J.E., C.J. Phene, R.B. Hutmacher, K.R. Davis, R.A. Schoneman, S.S. Vail and R.M. Mead. (1999). Subsurface drip irrigation of row crops: a review of 15 years of research at the Water Management Research Laboratory. Agricultural Water Management. 42:1-27. Elsevier.

Dastane, N.G. (1978). Efective Rainfall in Irrigated Agriculture. FAO Irrigation and Drainage Paper $N^{\circ}$ 25. FAO. Roma

Fereres, E., W.O. Pruitt, J.A. Beutel, D.W. Henderson, E. Holzapfel, H. Shulbach and K. Uriu. (1981). ET an drip irrigation scheduling, In drip irrigation management, of Fereres Ed. University of California. Div. of Agric. Sci. No 21259. pp. 8-13

Martínez, J. and Reca, J. (2014). "Water Use Efficiency of Surface Drip Irrigation versus an Alternative Subsurface Drip Irrigation Method." J. Irrig. Drain Eng., 10.1061/(ASCE)IR.19434774.0000745, 04014030 
AERYDXXIV Congreso Nacional de Riegos, Sevilla 2016

Orgaz, F. y E. Fereres. (1999). Riego, en el cultivo del olivo, de Barranco, Fernández y Rallo Eds. Mundi-Prensa. Madrid 